\title{
Comparação de métodos de amostragem para avaliação do sistema radicular da cana-de-açúcar
}

\author{
Comparison of sampling methods to evaluate sugarcane root system
}

\section{Adriana Marcela Silva-Olaya ${ }^{1}$; Carlos Eduardo Pellegrino Cerri²; Carlos Clemente Cerri ${ }^{3}$}

1 Docente. Ph.D. Universidad de la Amazonia, Florencia-Caquetá, Colombia, amsolayaa@gmail.

2 Docente. Ph.D. Departamento de Ciência do Solo, Escola Superior de Agricultura Luiz de Queiroz (ESALQ-USP), Piracicaba, SP, Brasil, cepcerri@usp.br.

3 Docente. Ph.D. Centro de Energia Nuclear na Agricultura (CENA-USP) Piracicaba, SP, Brasil.

Citação: SILVA-OLAYA, A.; PELLEGRINO, C.; CERRI, C. 2017. Comparação de métodos de amostragem para avaliação do sistema radicular da cana-de-açúcar. Rev. Cienc. Agr. 34(1):7-16. doi: http://dx.doi. org/10.22267/rcia.173401.59.

Recebido: Janeiro 22 de $2017 . \quad$ Aceito: Abril 19 de 2017.

\begin{abstract}
RESUMO
0 conhecimento da arquitetura do sistema radicular da cana-de-açúcar ainda é limitado devido às dificuldades metodológicas para o seu estudo. A forma correta de avaliar o sistema radicular não foi claramente estabelecida. Este estudo objetivou a comparação de dois métodos diretos de avaliação da biomassa radicular: coleta de monólitos de solo e coleta de amostras em sonda. Para isto foram estabelecidas três parcelas experimentais $\left(7,2 \mathrm{~m}^{2}\right)$ completamente ao acaso em área de plantio de cana-de-açúcar sobre solo Neossolo Quartzarenico localizado no município de São Pedro - SP. Uma planta dentro de cada parcela experimental foi selecionada para o estudo do sistema radicular. A amostragem com sonda foi realizada em 9 pontos dentro da metade do polígono de voronoi e a densidade radicular estimada em 9 combinações de pontos de amostragem. As amostras de raízes coletadas pelos dois métodos de estudo foram separadas do solo por meio da lavagem com água corrente em um conjunto de peneiras. Posteriormente as raízes foram colocadas para secar e finalmente pesadas em balança analítica. Diferenças significativas $(\mathrm{p}<0.05)$ foram encontradas para os resultados da densidade radicular entre as camadas avaliadas para cada tratamento. Tanto o método monólito quanto o método da sonda indicaram tendência de decréscimo da biomassa radicular com a profundidade. De forma geral o método da sonda tendeu a subestimar a quantidade de raiz encontrada na camada superficial. Somente duas combinações de amostragem com sonda mostraram ser equiparáveis ao método do monólito.
\end{abstract}

Palavras-chave: biomassa radicular, raiz, sonda, Saccharum officinarum. 


\begin{abstract}
The knowledge about the architecture of sugarcane root system is limited due to difficulties associated to methodological aspects. The correct way to assess the root system is still not clearly established. This study aimed to compare two direct methods of root biomass evaluation: soil monoliths and root sampling from probes. Three experimental plots $\left(7.2 \mathrm{~m}^{2}\right)$ were randomly established in an area under sugarcane planting on Typic Quartzipsamments located in São Pedro, Sao Paulo state, Brazil. One plant in each experimental plot was selected for root system assessment. The sampling with probes was performed in nine points inside half of the Voronoi polygon and the root density was estimated in nine combinations of sampling points. The root samples collected by the two methods were separate from soil particles by washing in a set of sieves. Afterwards, the roots were oven-dried and weighed. Significant differences $(p<0.05)$ in the density root were found among the soil layers in each treatment. A trend of decreasing root density with soil depth was found in both methods. The probes method tended to underestimate the amount of root found in the soil top layer. Only two sampling combinations with probe indicated to be equivalent to the monolith method.
\end{abstract}

Key words: probe, root, root biomass, Saccharum officinarum.

\section{INTRODUÇÃO}

A cana-de-açúcar tem sido considerada como uma das melhores opções dentre as matérias primas para produção de bioenergia. Isto tem acarretado substanciais impactos na área cultivada e na produtividade desta cultura. No ano 2009, a colheita de cana-de-açúcar no mundo resultou em cerca de quatro vezes o valor registrado em 1961, sendo que a área colhida passou de 8 a 23 milhões de hectares no mesmo período de tempo (Faostat, 2011).

O Brasil destaca-se como principal produtor de cana-de-açúcar, respondendo por mais de um terço da produção mundial. 0 último relatório da FAO indica que no ano 2014, foram colhidos 10,4 milhões de hectares de cana-de-açúcar no Brasil, com uma produção de 736 milhões de toneladas. Projeções futuras do setor sucroalcooleiro objetivam aumentar a produção de etanol nos próximos cinco anos para 58,8 bilhões de litros. Atingir essas metas nacionais implicará aumentar a área plantada com cana-de-açúcar para 19 milhões de hectares (CONAB, 2015). Nesse contexto, a definição de práticas de manejo que conduzam a melhoras na produtividade e sustentabilidade desta cultura tornam-se importantes ferramentas. O conhecimento do sistema radicular da cultura da cana-deaçúcar é um pré-requisito para a definição e uso adequado de técnicas agronômicas tais como o espaçamento, aplicação de adubos, irrigação, drenagem, controle de erosão, operações culturais entre outras (Casagrande, 1991).

Porém, apesar da importância da dinâmica do sistema radicular na interface solo-planta-água, poucos estudos têm sido realizados nessa área devido às dificuldades associadas a aspectos metodológicos e a complexidade do sistema radicular como um todo, o qual varia tanto no tempo quanto no espaço influenciado pelas condições físicas, químicas e biológicas do solo, além de caracteres fenotípicos. 
Todavia, a forma correta de avaliar o sistema radicular ainda não foi claramente estabelecida (Vasconcelos et al., 2003). Uma das técnicas mais comuns na estimativa da biomassa radicular é a utilização da relação fitomassa:raiz (Shoot:root), onde a massa seca do sistema radicular é calculada com base em medições da biomassa aérea (Bolinder et al., 1997; Campbell et al., 2000). No entanto, há muitas incertezas associadas a esta metodologia devido a considerável variabilidade encontrada nos dados.

Métodos diretos de avaliação nos quais amostras de raízes são coletadas em um determinado volume de solo vêm sendo amplamente utilizados. 0 método de amostragem em monólito é considerado como método padrão já que permite estimar maior quantidade de raízes, o que resulta em uma maior exatidão (Köpke, 1981). Porém a principal limitação desta técnica baseia-se no tempo gasto e no esforço físico que ela envolve.

A coleta de amostras em sonda é um método de amostragem que mostra-se como uma alternativa viável na avaliação do sistema radicular, uma vez que permite através de menor esforço quantificar a massa de raízes em um menor volume de solo. Otto et al. (2009) indicam esta metodologia como equiparável à metodologia do monólito, sem diferença significativa entre as duas. Entretanto, Vasconcelos et al. (2003) encontraram que a amostragem com sonda superestima a quantidade de raízes sugerindo que um maior número de amostras deve ser coletada quando esta técnica for utilizada.

Estudos in-situ não destrutivos, nos quais o crescimento radicular é acompanhado através de minirizotrons inseridos no solo são também utilizados nessa perspectiva (Ball-Coelho et al., 1992; Smit et al., 2000; Hendricks et al., 2006). A imprecisão dessa técnica de imagens esta associada à aplicação de fatores de correção na transformação de unidades de longitude para massa radicular.

A identificação de um método de amostragem para avaliação do sistema radicular que permita obter resultados confiáveis e que apresente facilidade operacional é um aspecto importante para o avanço de conhecimentos na área. Diante do exposto este estudo objetivou a comparação, no mesmo local e no mesmo período de tempo, de dois métodos diretos de amostragem para avaliação da biomassa radicular de cana-de-açúcar: Sondagem e monólitos de solo coletados no polígono de Voronoi, espaço elementar definido como a metade da distancia entre uma planta e as suas vizinhas. Nesta última metodologia, evita-se a coleta de grandes volumes de solo e considera-se que todas as raízes da planta amostrada que crescem fora do polígono são balanceadas pelas raízes da planta vizinha que cresce no interior do polígono traçado (Saint-André et al., 2005; Levillain et al., 2011).

\section{MATERIAIS E MÉTODOS}

0 estudo foi conduzido em área de plantio de cana-de-açúcar (primeiro ciclo) com 18 meses de idade, com a variedade RB86-7515 (RIDESA-UFV) colhida mecanicamente, em solo classificado como Neossolo Quartzarênico ortico (Santos et al., 2013) localizado no município de São Pedro, no nordeste do estado de São Paulo. O clima da região, de acordo com a classificação de Köppen, é Aw: tropical com verão chuvoso e inverno seco, caracterizado por precipitações 
menores do que $30 \mathrm{~mm}$ no mês mais seco e temperatura média do mês mais quente superior a $22^{\circ} \mathrm{C}$ e do mês mais frio inferior a $18^{\circ} \mathrm{C}$.

Três parcelas experimentais com dimensões de $2,4 \times 3 \mathrm{~m}\left(7,2 \mathrm{~m}^{2}\right)$ cada uma foram estabelecidas completamente ao acaso no local experimental. Análises com fins de caracterização físico-química do solo foram realizadas na área de estudo antes do estabelecimento do experimento (Tabela 1). Em cada uma das parcelas foi selecionada uma planta e posteriormente traçado o polígono de voronoi para cada uma delas, o qual corresponde ao espaço elementar definido como a metade da distancia entre uma planta e as suas vizinhas (Figura 1a). Nesse sistema todas as raízes da planta amostrada que crescem fora do polígono são balanceadas pelas raízes da planta vizinha que cresce no interior do polígono traçado (Saint-André et al., 2005; Levillain et al., 2011).

A massa de raiz seca e a distribuição do sistema radicular no perfil do solo foram avaliadas pelo método de coleta de amostras de solo em monólitos e sonda na área correspondente a metade do polígono de Voronoi (Figura 1a). Cada método de amostragem foi aplicado em uma planta por parcela, totalizando assim três repetições por método de amostragem.

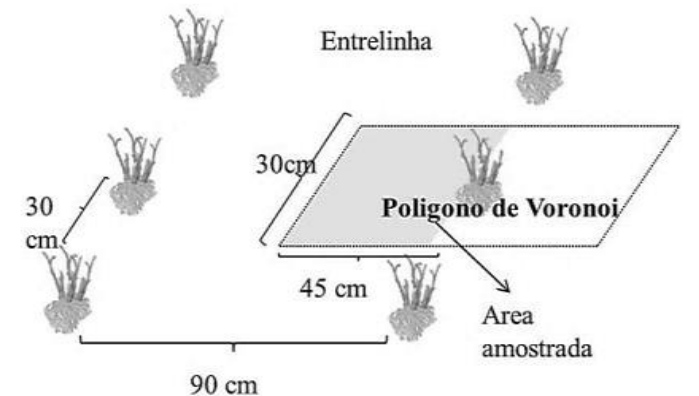

(a)

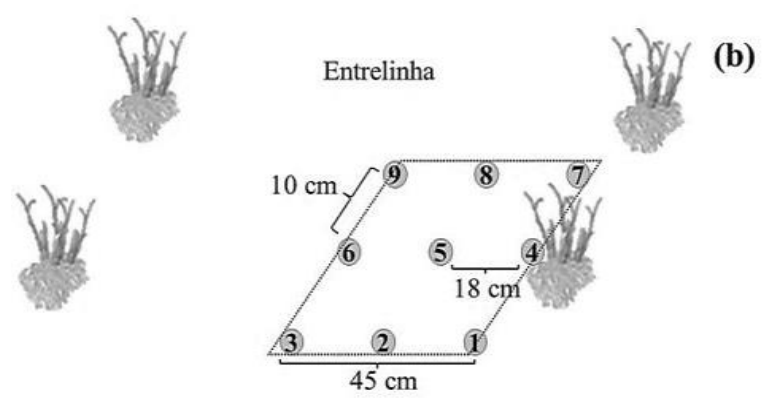

Figura 1. Esquema de amostragem da raiz na metade do polígono de Voronoi pelo método do monólito (a) e pelo método da sonda

(b) (Adaptado de Saint-andré, 2005).

Para a amostragem pelo método do monólito (M1) foi aberta uma trincheira de $1 \times 1 \times 1 \mathrm{~m}$ rente a planta a ser avaliada. Posteriormente, foram retirados monólitos de solo com dimensões de $0,45 \times 0,3 \times 0,2 \mathrm{~m}$ da parede da trincheira, até atingir $1 \mathrm{~m}$ de profundidade, totalizando 5 monólitos por parcela com volume de $0,027 \mathrm{~m}^{3}$ cada um (Saint-André et al., 2005; Levillain et al., 2011).

Tabela 1. Caracterização física e química do Neossolo quartzarenico ortico.

\begin{tabular}{|c|c|c|c|c|c|c|c|c|c|c|c|}
\hline \multirow{2}{*}{$\begin{array}{c}\text { Profundidade } \\
\text { cm }\end{array}$} & pH & $\mathbf{P}$ & $\mathbf{K}$ & $\mathrm{Ca}$ & Mg & Al & \multirow[t]{2}{*}{$\mathrm{T}$} & \multirow{2}{*}{$\begin{array}{l}\mathrm{V} \\
\%\end{array}$} & Areia & Argila & Silte \\
\hline & & $\mathrm{mg} \mathrm{kg}^{-1}$ & \multicolumn{4}{|c|}{----- mmolc kg-1 ------ } & & & \multicolumn{3}{|c|}{-----g kg-1 ----- } \\
\hline $0-20$ & 5,7 & 63 & 0,6 & 11 & 2 & 4 & 34,7 & 39 & 891 & 50 & 59 \\
\hline $20-40$ & 5,0 & 19 & 0,2 & 5 & 1 & 6 & 27,5 & 23 & 845 & 50 & 105 \\
\hline $40-60$ & 4,9 & 2 & $<0,2$ & 3 & $<1$ & 8 & 25,8 & 15 & 843 & 75 & 82 \\
\hline $60-80$ & 4,7 & 2 & $<0,2$ & 3 & $<1$ & 8 & 20,3 & 18 & 843 & 100 & 57 \\
\hline $80-100$ & 4,6 & 1 & $<0,2$ & 2 & $<1$ & 8 & 20,6 & 15 & 847 & 100 & 53 \\
\hline
\end{tabular}


A coleta de amostras pelo método da sonda foi realizada utilizando-se sondas de aço inoxidável de 1 metro de comprimento com 0,055m de diâmetro interno. A amostragem foi executuda em 9 pontos no interior da metade do polígono de Voronoi os quais estiveram posicionados a diferentes distâncias da planta objeto de estudo (Figura 1b) a fim de identificar a combinação de amostragem que permita obter resultados equiparáveis aos obtidos pelo método padrão do monólito.

A sondagem foi realizada a cada $20 \mathrm{~cm}$ até atingir profundidade máxima de $1 \mathrm{~m}$. Desta forma $0,48 \mathrm{dm}^{3}$ de solo foram coletados em cada camada avaliada, totalizando $2,38 \mathrm{dm}^{3}$ por ponto amostrado.

As amostras de raízes coletadas pelos dois métodos de estudo foram separadas do solo pela lavagem com jato de água corrente em um conjunto de peneiras de 5,2 e $1 \mathrm{~mm}$. Posteriormente as amostras foram secas em estufa a $60^{\circ} \mathrm{C}$ até a obtenção de massa constante, e finalmente pesadas em balança analítica para a determinação da massa de matéria seca de raízes em cada uma das camadas amostradas.

A densidade radicular foi estimada em cada uma das parcelas, para cada avaliada. No método da sonda, a densidade foi estimada considerando-se nove combinações de pontos de amostragem: Densidade radicular média dos nove pontos (D1); densidade radicular média dos pontos 1, 2, 3 (D2); densidade radicular média dos pontos 4,5 , 6 (D3); densidade radicular média dos pontos 7, 8, 9 (D4); densidade radicular média dos pontos 3, 5, 7 (D5); densidade radicular média dos pontos 1, 5,9 (D6); densidade radicular média dos pontos 2, 5, 8 (D7); densidade radicular média dos pontos 1,4 , 7 (D8); densidade radicular média dos pontos 3, 6, 9 (D9).

Os resultados obtidos foram submetidos ao teste $\mathrm{F}$ de análise da variância. As médias da densidade radicular estimadas para cada tratamento foram comparadas através do teste de Tukey ao nível de significância de $\mathrm{p}<0,05$.

\section{RESULTADOS E DISCUSSÃO}

A análise de caracterização físico-química do solo indica a textura arenosa do solo (Santos et al, 2013) de baixa fertilidade, com valores de saturação por bases inferiores a $25 \%$ nas camadas subsuperficiais, onde a maior parte da CTC do solo está ocupada por alumínio (Tabela 1).

Somente na camada superficial os teores de cálcio e fósforo foram relativamente altos, devido as operações de calagem e adubação realizadas como parte do manejo da cultura. Esta característica influenciou o desenvolvimento do sistema radicular das plantas, uma vez que a maior densidade radicular foi observada nessa camada, com uma tendência de decréscimo ao longo do perfil do solo (Figura 2).

Vasconcelos e Garcia (2005) expressam que o cálcio é essencial para a divisão celular e para a funcionalidade da membrana celular. As raízes apresentam tendência natural de maior desenvolvimento em zonas com maior disponibilidade de nutrientes. Deficiências de potássio e fósforo podem causar redução na massa radicular, além de mudanças no volume e distribuição lateral do sistema radicular (Hacckett, 1968). 


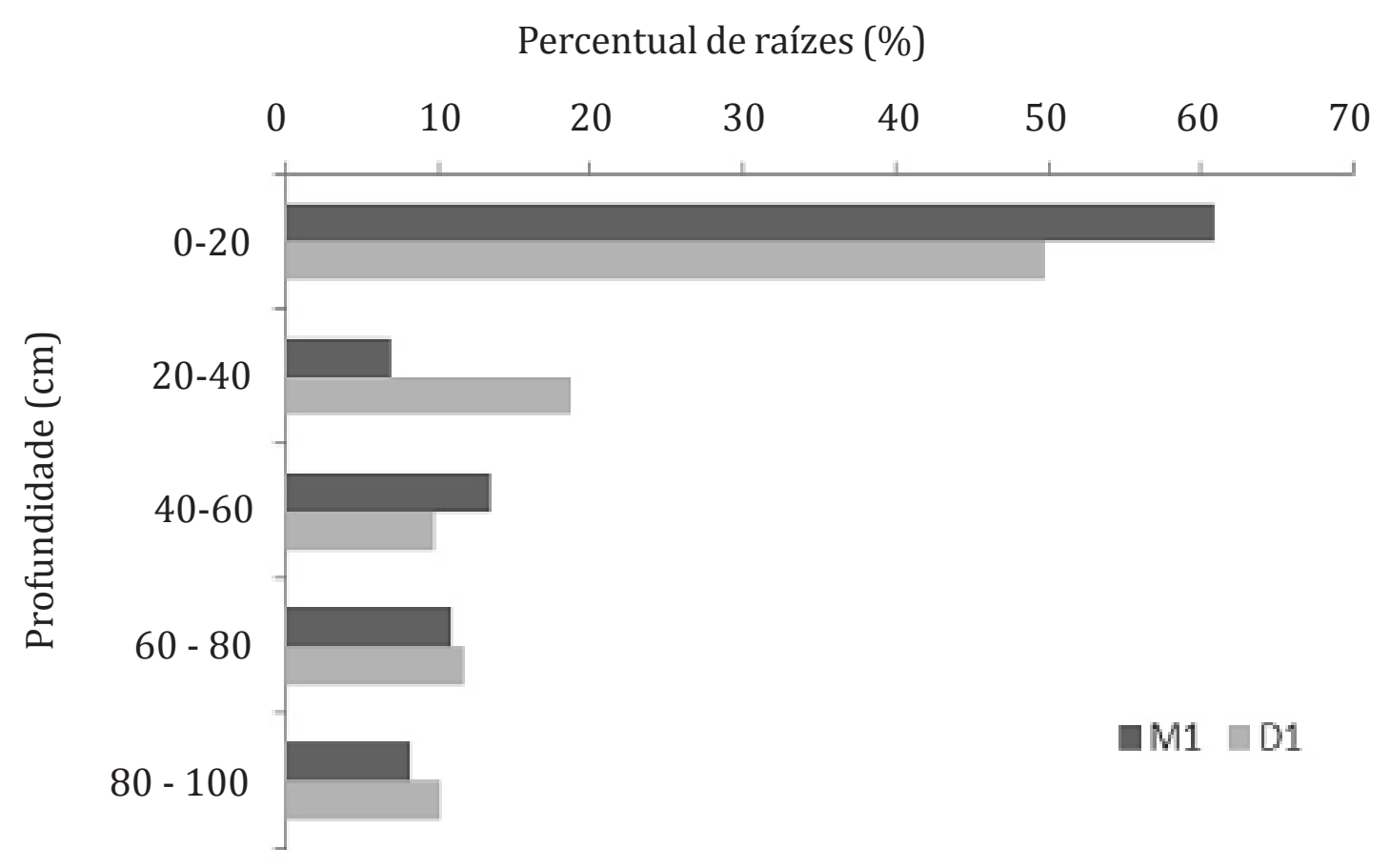

Figura 2. Distribuição percentual do sistema radicular ao longo do perfil do solo pelo método do monólito (M1) e da sonda (D1).

A profundidade do solo foi um fator que influenciou a quantidade de raízes. Diferenças significativas $(\mathrm{p}<0,05)$ foram encontradas na densidade radicular entre as camadas avaliadas para cada tratamento (Tabela 2). No método do monólito $61 \%$ da biomassa radicular foi encontrada entre $0-20 \mathrm{~cm}$ de profundidade decrescendo até $8 \%$ na camada mais profunda $(80-100 \mathrm{~cm}) .0$ método da sonda indicou tendência semelhante, com $50 \%$ da biomassa radicular na camada superficial $(0-20 \mathrm{~cm})$ e $10 \%$ na camada de $80-100 \mathrm{~cm}$ de profundidade (Figura 2).

Vários trabalhos têm apresentado dados de distribuição radicular no perfil do solo semelhantes aos encontrados nesta pesquisa. Evans (1938) e Alvarez et al., (2000) encontraram biomassa radicular variando entre $70-74 \%$ na camada de $0-30 \mathrm{~cm}$ e $70-85 \%$ na camada de 0-40 cm de profundidade, respectivamente.
Faroni e Trivelin (2006) avaliaram o sistema radicular da cana soca em diferentes épocas e encontraram que, 12 meses após a colheita, $64 \%$ das raízes quantificadas até $80 \mathrm{~cm}$ de profundidade situavam-se nos primeiros $20 \mathrm{~cm}$ do solo. Entretanto, Ball-Coelho et al., (1992) reportou distribuição radicular em superfície menor à encontrada neste trabalho, variando de 62 a $69 \%$ na camada de $0-50 \mathrm{~cm}$ de profundidade.

A densidade média de raízes considerando $100 \mathrm{~cm}$ de profundidade foi relativamente superior a encontrada em outros trabalhos (Ball-Coelho et al., 1992; Alvarez et al., 2000; Vasconcelos et al., 2003; Faroni y Trivelin, 2006; Otto et al., 2009), com valores de 0,8g $\mathrm{dm}^{3}$ pelo método do monólito e $0,5 \mathrm{~g} \mathrm{dm}^{3}$ no método de amostragem com sonda (D1). As características físico-químicas do local de estudo em combinação com fatores fenotípicos podem ter influenciado esse resultado. 
Tabela 2. Densidade radicular da cana-de-açúcar pelo método do monólito (M1) e da sonda (D1, D2, D3, D4 , D5, D6, D7 D8, D9) em cinco profundidades.

\begin{tabular}{|c|c|c|c|c|c|}
\hline $\begin{array}{c}\text { Métodos de } \\
\text { amostragem } \\
\text { (Combinaçóes } \\
\text { de pontos) }\end{array}$ & $0-20 \mathrm{~cm}$ & 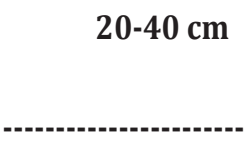 & ----g dm & $60-80 \mathrm{~cm}$ & $80-100 \mathrm{~cm}$ \\
\hline M1 & $2,49 \pm 0,17 \mathrm{Aa}^{*}$ & $0,28 \pm 0,05 \mathrm{Ac}$ & $0,55 \pm 0,16 \mathrm{Ab}$ & $0,44 \pm 0,15 \mathrm{Abc}$ & $0,33 \pm 0,09 \mathrm{Ac}$ \\
\hline D1 & $1,28 \pm 0,32 \mathrm{Ba}$ & $0,48 \pm 0,27 \mathrm{Ab}$ & $0,25 \pm 0,05 \mathrm{Ab}$ & $0,30 \pm 0,06 \mathrm{Ab}$ & $0,26 \pm 0,03 \mathrm{Ab}$ \\
\hline D2 & $1,19 \pm 0,27 \mathrm{Ba}$ & $0,71 \pm 0,46 \mathrm{Aab}$ & $0,35 \pm 0,02 \mathrm{Ab}$ & $0,35 \pm 0,02 \mathrm{Ab}$ & $0,32 \pm 0,09 \mathrm{Ab}$ \\
\hline D3 & $1,36 \pm 0,39 \mathrm{Ba}$ & $0,31 \pm 0,11 \mathrm{Ab}$ & $0,25 \pm 0,10 \mathrm{Ab}$ & $0,31 \pm 0,12 \mathrm{Ab}$ & $0,21 \pm 0,05 \mathrm{Ab}$ \\
\hline D4 & $1,28 \pm 0,50 \mathrm{Ba}$ & $0,43 \pm 0,23 \mathrm{Ab}$ & $0,14 \pm 0,03 \mathrm{Ab}$ & $0,23 \pm 0,10 \mathrm{Ab}$ & $0,23 \pm 0,12 \mathrm{Ab}$ \\
\hline D5 & $1,82 \pm 0,58 \mathrm{Aa}$ & $0,48 \pm 0,18 \mathrm{Ab}$ & $0,26 \pm 0,15 \mathrm{Ab}$ & $0,40 \pm 0,19 \mathrm{Ab}$ & $0,31 \pm 0,07 \mathrm{Ab}$ \\
\hline D6 & $1,03 \pm 0,39 \mathrm{Ba}$ & $0,54 \pm 0,45 \mathrm{Aa}$ & $0,29 \pm 0,14 \mathrm{Aa}$ & $0,41 \pm 0,10 \mathrm{Aa}$ & $0,26 \pm 0,10 \mathrm{Aa}$ \\
\hline D7 & $1,36 \pm 0,61 \mathrm{Ba}$ & $0,39 \pm 0,19 \mathrm{Ab}$ & $0,34 \pm 0,03 \mathrm{Ab}$ & $0,47 \pm 0,08 \mathrm{Aab}$ & $0,30 \pm 0,06$ Аа \\
\hline D8 & $1,56 \pm 0,35 \mathrm{Aa}$ & $0,83 \pm 0,71 \mathrm{Aab}$ & $0,26 \pm 0,12 \mathrm{Ab}$ & $0,33 \pm 0,09 \mathrm{Ab}$ & $0,34 \pm 0,10 \mathrm{Ab}$ \\
\hline D9 & 0,91 $\pm 0,13 \mathrm{Ba}$ & $0,20 \pm 0,01 \mathrm{Ab}$ & $0,15 \pm 0,02 \mathrm{Ac}$ & $0,10 \pm 0,04 \mathrm{Ac}$ & $0,12 \pm 0,07 \mathrm{Abc}$ \\
\hline
\end{tabular}

"Valores seguidos pela mesma letra maiúscula na coluna e mesma letra minúscula na linha não diferem entre se pelo teste de Tukey ao nível de 5\% de significância.

A distribuição e penetração das raízes são afetadas pela densidade, temperatura, teor de água, aeração, pH, quantidade e distribuição de nutrientes, entre outras características do solo (Allmaras et al., 1988). Abayomi (1989) estudando os efeitos do tipo de solo e ciclo de cultivo sobre o desenvolvimento radicular encontrou que a massa de raízes de cana planta sobre solo argilosso correspondeu a $17 \%$ da massa radicular encontrada em solo com alto teor de areia, sendo que essa porcentagem aumenta para 59\% na cana soca.

Quando comparados os métodos de estudo do sistema radicular verificaram-se diferenças significativas pelo teste de Tukey a $5 \%$ entre os métodos na camada de 0-20 de profundidade (Tabela 2), sendo que nas outras camadas tais diferenças não foram detectadas.

De forma geral o método da sonda tendeu a subestimar a quantidade de raiz encontrada na camada superficial. Somente as combinações de amostragem com sonda D5 e D8 mostraram ser equiparáveis ao método do monólito, sem diferença significativa entre os métodos em nenhuma das profundidades estudadas. Porém, a amostragem com sonda na combinação D5 parece ter melhor equivalência na quantidade de raízes em relação ao método do monólito, uma vez que mostrou valores sistematicamente mais próximos do que os observados em D8.

Na camada de $20-40 \mathrm{~cm}$ de profundidade ainda sem diferença significativa entre os tratamentos, o método da sonda tendeu a superestimar a densidade radicular em relação ao monólito (Figura 2). Este comportamento coincide com os resultados reportados por Vasconcelos et al. (2003) e Faroni (2004), onde a amostragem com sonda apresentou alto coeficiente de variação, fato que incidiria nesse resultado segundo os autores. 
Otto et al. (2009) avaliando o efeito da adubação nitrogenada sobre o sistema radicular da cana-de-açúcar encontraram que a densidade radicular média até $60 \mathrm{~cm}$ de profundidade estimada pelo método da sonda não foi diferente da determinada através do método do monólito. Porém quando avaliada a distribuição da raiz no perfil do solo, encontrou-se que o método da sonda não permitiu a detecção de diferenças significativas na massa de raízes entre os tratamentos, tal como foi evidenciado no método do monólito. Os resultados encontrados nesta pesquisa sugerem que a coleta de amostras de solo com sonda nos pontos indicados na combinação de amostragem D5 poderia ser usada em estudos que visam à avaliação da arquitetura do sistema radicular em solos com características físicas semelhantes, facilitando as operações de amostragem e diminuindo por tanto o tempo gasto nas mesmas em comparação ao método do monólito.

Com o intuito de aumentar a precisão da técnica e garantir resultados confiáveis, a amostragem poderia se expandir à outra metade do polígono de Voronoi, seguindo os mesmos distanciamentos dos pontos de coleta em relação à planta avaliada. Entretanto, pesquisas que requerem estimativas ainda mais precisas devem considerar a utilização de métodos de amostragem que explorem maior volume de solo e permitam a quantificação da massa do rizoma, cuja estimativa não é possível com o método da sonda.

Sob condições adequadas de cultivo, a canade-açúcar desenvolve raízes que podem se estender profundamente, conferindo à planta capacidade de suportar escassez de água (Inman-Bamber e Smith, 2005). Assim, o conhecimento do comportamento do sistema radicular da cana-de-açúcar é fundamental na identificação de práticas de manejo que contribuam a incrementos na produtividade.

\section{CONCLUSÃO}

Uma vez comparado os dois métodos de amostragem, verificou-se quando utilizado método da sonda, a posição do ponto de amostragem define o grau de precisão dos resultados obtidos, sendo que na maioria das situações a sondagem tende a subestimar a densidade radicular da cana-de-açúcar na camada de 0 - $20 \mathrm{~cm}$ de profundidade e superestimar tal variável na camada de $20-40 \mathrm{~cm}$. A coleta de amostras com sonda seguindo a combinação de amostragem D5 forneceu resultados equiparáveis aos encontrados com método do monólito, porém trabalhos adicionais seriam necessários em solos sob outras caraterísticas físico-químicas.

\section{AGRADECIMENTOS}

Os autores agradecem a Fundação de Amparo à Pesquisa do Estado de São Paulo (FAPESP) pelo financiamento do projeto, e à Coordenação de Aperfeiçoamento de Pessoal de Nível Superior (CAPES) pela concessão da bolsa do primeiro autor.

\section{REFERENCIAS BIBLIOGRÁFICAS}

ABAYOMI, Y.A. 1989. Effect of soil type and crop cycle on root development and distribution pattern of a commercial sugarcane cultivar under normal irrigation and field conditions at Bacita Estate, Nigeria. Turrialba. Scientific Research. 39: 78-84.

ALLMARAS, R. R.; KRAFT, J. M.; MILLER, D. E. 1988. Effects of soil compaction and incorporated 
crop residue on root health. Annual Review Phytopathology. 26: 219-243.

ALVAREZ, I. A.; CASTRO, P. D. C.; NOGUEIRA, M. C. S. 2000. Crescimento de raízes de cana crua e queimada em dois ciclos. ScientiaAgricola. 57:653659. Doi: 10.1590/S0103-90162000000400009.

BALL B.; SAMPAIO, E. V. S. B.; TIESSEN, H.; STEWART, J. W. B. 1992. Root dynamics in plant and ratoon crops of sugar-cane. Plant and Soil. 142: 297-305. doi: 10.1007/BF00010975.

BOLINDER, M. A.; ANGERS, D. A.; DUBUC, J. P. 1997. Estimating shoot to root ratios and annual carbon inputs in soils for cereal crops. Agriculture Ecosystems \& Environment. 63: 6-66. Doi: 10.1016/S0167-8809(96)01121-8.

CAMPBELL, C. A.; ZENTNER, R. P.; LIANG, B. C.; ROLOFF, G.; GREGORICH, E. C.; BLOMERT, B. 2000. Organic $C$ accumulation in soil over 30 years in semiarid southwestern Saskatchewan - Effect of crop rotations and fertilizers. Canadian Journal of Soil Science. 80: 179-192. Doi: 10.4141/S99-028.

CASAGRANDE, A. A. 1991. Topicos de morfologia e fisiologia da cana-de-açucar. Funep. Jaboticabal. $157 p$.

CONAB CNDA. 2015. Acompanhamento da safra brasileira Cana-de-açúcar. Safra 2014/2015. Ed. Agronegócio Ddpaei-Sdid, CONAB. Brasilia. 1-29p.

EVANS, H. 1938. Studies on the Absorbing Surface of Sugar-cane Root Systems I. Method of Study with Some Preliminary Results. Annals of botany. 2: 159-182.

FAOSTAT. 2011. Food and agriculture organization of the united nations. 2011. Em: http://faostat.fao.org/; consulta: julio, 2011.

FARONI, C.E.; TRIVELIN, P.C.O. 2006. Quantificação de raízes metabolicamente ativas de canade-açúcar. Pesquisa Agropecuaria Brasileira. 41: 1007-1013.

FARONI, C.E. 2004. Sistema radicular da cana-deaçúcar e identificação de raízes metabolicamente ativas. Em: http://www.teses.usp.br/teses/dis- poniveis/11/11140/tde.../carlos.pdf; consulta: julio, 2011.

HACCKETT, C. 1968. A study of the root system of barley. I effects of nutrition on two varieties. New Phytologist.67: 287-299. doi: 10.1111/j.14698137.1968.tb06384.x.

INMAM-BAMBER, N.G.; SMITH, D.M. 2005. Water relations in sugarcane and response to water deficits. Field Crops Research. 92: 185-202.

HENDRICKS, J. J., HENDRICK, R. L., WILSON, C. A., MITCHELL, R. J., PECOT, S. D.;GUO, D. 2006. Assessing the patterns and controls of fine root dynamics: an empirical test and methodological review. Journal of Ecology. 94: 40-57. doi: 10.1111/j.1365-2745.2005.01067.x.

KÖPKE, V. 1981. Methods for studyng root growth. 303 - 318p. En: SYMPOSIUM ON THE SOIL/ROOT SYSTEM, 1, Londrina, 1980. Proceedings. Londrina, Fundação Instituto Agronômico do Paraná.

LEVILLAIN, J.; M'BOU, A. T.; DELEPORTE, P.; SAINT, L.; JOURDAN, C. 2011. Is the simple auger coring method reliable for below-ground standing biomass estimation in Eucalyptus forest plantations?. Annal of botany. 108: 221-230. doi: 10.1093/aob/ mcr102.

OTTO, R.; TRIVELIN, P. C. O.; FRANCO, H. C. J.; FARONI, C. E.; VITTI, A. C. 2009. Root system distribution of sugar cane as related to nitrogen fertilization, evaluated by two methods: monolith and probes. Revista Brasileira de Ciência do Solo. 33: 601-611.

SAINT L.; M'BOU, A. T.; MABIALA, A.; MOUVONDY, W.; JOURDAN, C.; ROUPSARD, O.; NOUVELLON, Y. 2005. Age-related equations for above- and below-ground biomass of a Eucalyptus hybrid in Congo. Forest Ecology and Management. 205: 199-214. doi: 10.1016/j.foreco.2004.10.006.

SANTOS, H.G.; JACOMINE, P.K.T.; ANJOS, L.H.C.; OLIVEIRA, V.A.; LUMBRERAS, J.F.; COELHO, M.R.; ALMEIDA, J.A.; CUNHA, T.J.F.; OLIVEIRA, J.B. 2013. de. Sistema brasileiro de classificação de solos. Rev. e Ampl. Brasília: Embrapa. 353 p 
SMIT, A. L.; GEORGE, E.;GROENWOLD, J. 2000. Root observations and measurements at (transparent) interfaces with soil. 235-271P. En: SMIT A.L.; BENGOUGH A.G.; ENGELS, C.; VAN NOORDWIJK, M.; PELLERIN, S.; VAN DE GEIJN S.C.; EDS. Root Methods: A Hand Book. SpringerBerlin Heidelberg, Berlin. 581 p.

VASCONCELOS, A. C. M.; CASAGRANDE, A. A.; PERECIN, D.; JORGE, L. A. C.; LANDELL, M. G. A. 2003. Avaliação do sistema radicular de cana-de-açúcar por diferentes métodos. Revista Brasileira de Ciência do Solo. 27: 849-858.

VASCONCELOS, A. C. M.; GARCIA, J. C. 2005. Desenvolvimento radicular da cana-de-açúcar. Canade-açúcar: Ambientes de produção. Informações Agronômicas. 110: 1-5. 